\title{
The contribution of backbone electrostatic repulsion to DNA mechanical properties is length scale dependent
}

\author{
Shiyan Xiao, ${ }^{\dagger, \ddagger}$ Haojun Liang, ${ }^{*, \dagger}$ and David J. Wales ${ }^{*, \ddagger}$ \\ $\dagger$ †AS Key Laboratory of Soft Matter Chemistry, Collaborative Innovation Center of \\ Chemistry for Energy Materials, Department of Polymer Science and Engineering, Hefei \\ National Laboratory for Physical Sciences at Microscale, University of Science and \\ Technology of China, Hefei, Anhui 230026, P. R. China \\ $\ddagger$ Department of Chemistry, University of Cambridge, Lensfield Road, Cambridge CB2 \\ $1 E W$, United Kingdom \\ E-mail: hjliang@ustc.edu.cn; dw34@cam.ac.uk
}




\begin{abstract}
The mechanics of DNA bending is crucially related to many vital biological processes. Recent experiments reported anomalous flexibility for DNA on short length scales, calling into doubt the validity of the harmonic worm-like chain (WLC) model in this region. In the present work, we systematically probed the bending dynamics of DNA at different length scales. In contrast to the remarkable deviation from the WLC description for DNA duplexes of less than three helical turns, our atomistic studies indicate that the neutral "null isomer" behaves in accord with the ideal elastic WLC, and exhibits a uniform decay for the directional correlation of local bending. The backbone neutralization weakens the anisotropy in the effective bending preference and the helical periodicity of bend correlation that have previously been observed for normal DNA. The contribution of electrostatic repulsion to stretching cooperativity and the mechanical properties of DNA strands is length scale dependent; the phosphate neutralization increases the stiffness of DNA below two helical turns, but decreases for longer strands. We find that DNA rigidity is largely determined by base pair stacking, with electrostatic interactions contributing only around $10 \%$ of the total persistence length.
\end{abstract}

\title{
Graphical TOC Entry
}

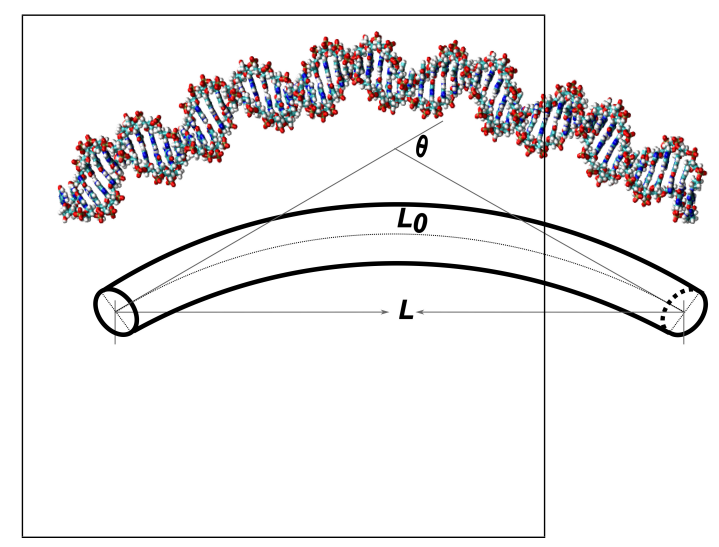

\section{Keywords}

American Chemical Society, $\mathrm{HTE}_{\mathrm{E}} \mathrm{X}$ 


\section{Introduction}

The mechanical properties of DNA play a key role for its activity in cells, and quantifying the intrinsic elasticity in the sub-persistence length $\left(l_{p} \approx 150\right.$ base pairs) is essential to understand the structural dynamics of cellular processes, such as replication and transcription. ${ }^{1,2}$ Previous experimental and theoretical investigations at the single molecule level suggested that DNA can be characterized as an ideal elastic rod, and well described by the traditional worm-like chain (WLC) model. ${ }^{3-5}$ However, this result has been challenged by recent experiments that probe the inherent flexibility and bending of DNA fragments on short length scales. The cyclization assays of around 100 base pairs (bp) DNA, based on ligation reaction, performed by Cloutier and Widom, revealed a much higher rate of cyclization than the prediction of the WLC model, indicating greatly enhanced flexility. ${ }^{6,7}$ This unexpected result is supported by later atomic force microscopy (AFM) and small-angle x-ray scattering experiments, ${ }^{8-10}$ and verified by the recent cyclization experiments of single DNA molecules directly monitored using single-molecule fluorescence resonance energy transfer (smFRET). ${ }^{11}$ Molecular dynamics (MD) simulations by Noy and Golestanian revealed a smooth crossover behaviour for the twist modulus, indicating less resistance to twisting for length scales less than about half a turn of the helix. ${ }^{12}$ This surprising length scale dependence questions the validity of the WLC model for DNA shorter than $l_{p}$. To explain the relatively large flexibility of short DNA, extended WLC models have been developed by allowing for the formation of temporary bubbles ${ }^{13}$ or kinks. ${ }^{14-17}$ However, there is still no reliable evidence for DNA segments with opened base pairs. ${ }^{18}$ Recent theoretical $^{19,20}$ and experimental studies $^{21,22}$ questioned the anomalous bending of DNA on short length scales, arguing that DNA can be well described by the harmonic WLC model. Although recent AFM experiments demonstrated that the bending fluctuation in DNA is well described as a WLC chain on all length scales beyond three helical turns ${ }^{21}$ the length limit at which the WLC model holds remains uncertain, and the mechanical properties of DNA at short lengths remain intriguing, controversial, and important. ${ }^{23-25}$ 
In this communication, we try to determine when DNA starts to deviate from the WLC model, and to uncover the origin of this deviation, using atomistic MD simulations of the intrinsic bending of dinucleotides to five helical turns. The rigidity of DNA is found to come from the compressive base stacking combined with the significant electrostatic repulsion along the backbone. To extract the electrostatic contribution from the overall effects, a "null isomer" of DNA is constructed, which is a hypothetical structure ${ }^{26-29}$ with the backbone neutralized by reducing the charge of the phosphate groups. ${ }^{26,29}$ Hence we quantitatively analyse the role of electrostatics in regulating the elasticity of DNA, structural stability and functional dynamics of DNA on intermediate length scales, especially the excessive flexibility. ${ }^{23,30}$

\section{Results and discussion}

The DNA sequence used in the experiments of Vafabakhsh and $\mathrm{Ha}^{11}$ is chosen as the model system, modified to ensure that no less than five copies of the 10 unique dinucleotide steps are included (GCTAG TACCG CAATA TAGAC TCGCT TCTAA TTGAC CATGA CTATC CTCAC CGCCA CCGTT GCG, see Supporting Information). The "null isomer" can be obtained by reducing backbone charge or introducing site-specific neutralizing chemical substitutions of phosphate oxygens. ${ }^{26-28}$ To avoid the the stereospecific effects induced by the incorporation of methylphosphonate or other groups ${ }^{27}$ such as bending orientation, groove width and water distribution, we neutralized a given phosphate group by adding charge of $+1.0|e|$, including one phosphorus $(\mathrm{P})$ and four oxygen (O1P, O2P, O3', O5') atoms, leaving the partial charges of all the other atoms of the nucleotides unchanged. The DNA geometric variables and helical parameters between two neighbouring base pairs are calculated using our in-house program package based on the methodology of 3DNA. ${ }^{31-33}$ According to the principles of the WLC model, the bending angle $\theta$ of two tangent vectors $\mathbf{z}_{i} \cdot \mathbf{z}_{j}$ separated by a distance $L_{i, j}$ obeys the general theory of fluctuations, ${ }^{34}\left\langle\cos \theta_{i, j}\right\rangle=\mathrm{e}^{-L_{i, j} / l_{p}}$, where $l_{p}$ 
is the bending persistence length. For a sufficiently weak bending rod, this equation can be rewritten in a linear form as $\left\langle\cos \theta_{i, j}\right\rangle \cong 1-\frac{1}{2}\left\langle\theta_{i, j}^{2}\right\rangle=1-L_{i, j} / l_{p}$. The apparent persistence length $l_{p}$ can then be derived using the growth rate of the averaged $\left\langle\cos \theta_{i, j}\right\rangle$ with respect to the fragment length $L_{i, j}$. The present analysis of the effects of backbone neutralization on the elastic properties of DNA at different fragments lengths was inspired by the previous contribution of Noy and Golestanian. ${ }^{12}$

Figure 1 presents the probability distribution $(P)$ of bending angles $(\theta)$ in various lengths of DNA. For normal DNA with segments longer than 25 base pairs, the MD derived distributions of $\ln P$ vary near linearly as $1-\cos \theta$, shown the gray dashed line in Figure 1c. This behaviour agrees very well with the WLC model, and agrees with the previous experimental and simulation results. ${ }^{21,35,36}$ On short length scales, a remarkable deviation from the WLC description is observed. In contrast to the length-dependent behaviour of the probability distribution for DNA bending, the "null isomer" DNA double helix with the same sequence but no net charge exhibits a completely different pattern, in which the MD distributions give descending straight lines of $\ln P$ versus $1-\cos \theta$ for all the DNA chains investigated. This linear dependence of ln $P$ on $1-\cos \theta$ is in good agreement with the WLC theory, and therefore reveals a homogeneous characteristic of bending fluctuations for neutral DNA. These results demonstrate that macroscopic elastic theory can accurately describe DNA bending at the base pair level if the net charge is removed.

As suggested by molecular simulations and statistical analysis of protein-DNA structures, ${ }^{35,37}$ the nonlinear behaviour of normal DNA on short length scales originates from the heterogeneity of the intrinsic geometric frustration in the B-DNA structure, such as the backbone compression, ${ }^{35,38}$ accompanied by anisotropy of groove bending. ${ }^{35,37}$ The anisotropy in the effective bending preference into the major or minor grooves, in addition to the sequence inhomogeneity of DNA, are responsible for the variations in local bending motions. ${ }^{39,40}$ These effects explain the significant downward deviation of the tails in the $\ln P$ curves with respect to the linear regression lines obtained for small angles for normal DNA fragments of 


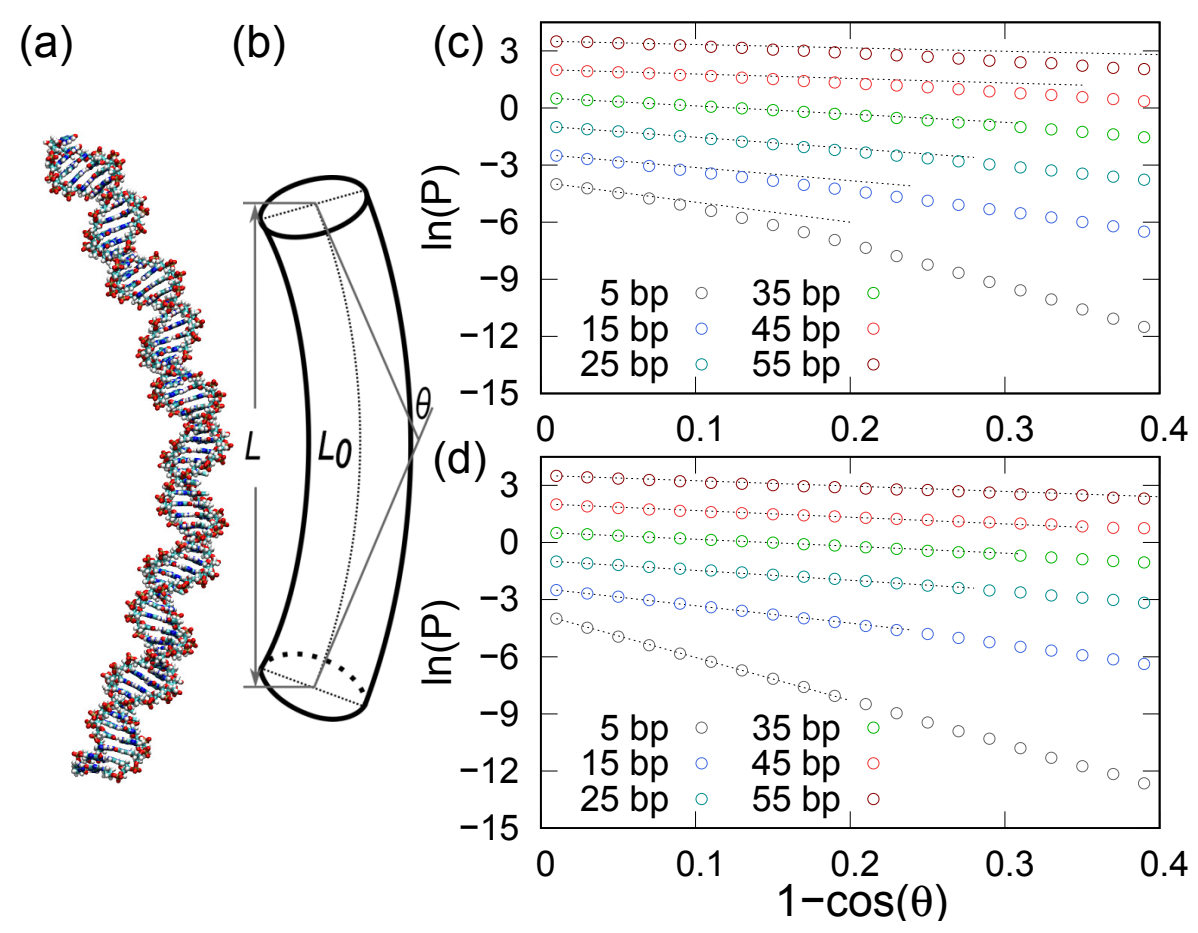

Figure 1: Probability distribution for bending angles $(\theta)$ in DNA fragments of $N=5,15,25,35,45$, and 55 bp, in normal DNA (c), and neutral DNA (d), in which backbone phosphates are neutralized. The gray dashed lines represent linear regression analysis of the initial points (at least five.). All plots are consecutively shifted vertically for clarity. After shifting, the values of the $\ln$ probabilities at $1-\cos \theta=0.01$ are $-4.0,-2.5,-1.0,+0.5,+2.0,+3.5$ for DNA fragments of $N=5,15,25,35,45$, and $55 \mathrm{bp}$, respectively. (a) $63 \mathrm{bp}$ DNA oligomer; (b) schematic diagram of the DNA contour length $\left(L_{0}\right)$, end-to-end distance $(L)$, and bending angle. 
less than two helical turns, shown in Figure 1c. The shorter the fragments, the larger the deviation from the WLC distribution derived using $l_{p}$ fitted for longer length scale. This anharmonic elastic behaviour will be masked for DNA beyond more than two or three helical turns because of averaging, therefore approaching a WLC angular distribution. Accordingly, the backbone electrostatic repulsion can be viewed as a significant perturbation to the elastic energy function of the WLC model, causing deviations from WLC theory for short DNA fragments. The reduction of backbone electrostatic repulsion decreases the perturbation coming from the neighbouring base pairs, and therefore reduces the bending anisotropy (Figure 1d). Our results indicate that the reduction of phosphate net charge helps to reduce the overall preference of bending direction for short DNA fragments, and therefore results in consistent bending behaviour on different length scales.

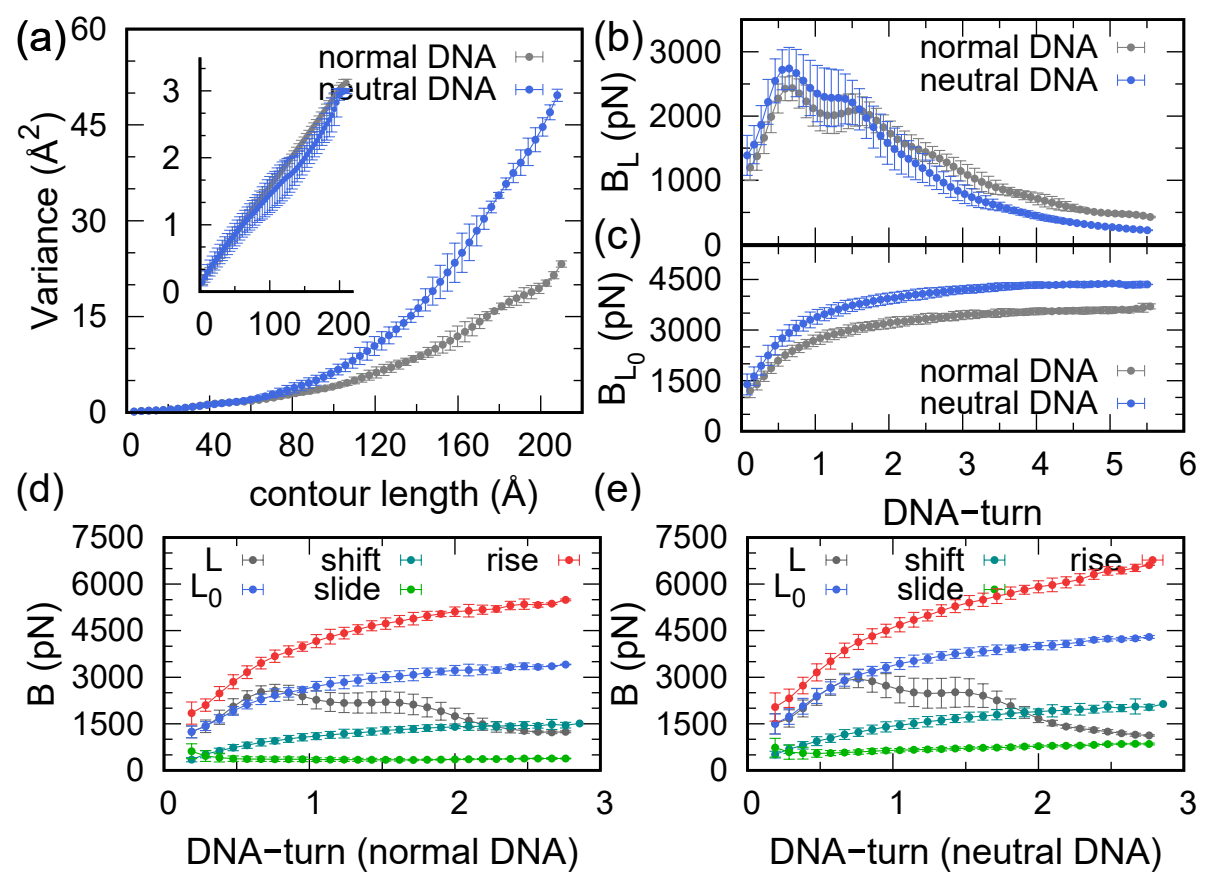

Figure 2: (a) The variance of the end-to-end distance $(L)$ and the contour length $\left(L_{0}\right.$, inset) extracted from MD simulations of $63 \mathrm{bp}$ DNA. Panels (a) and (c), the stretch modulus associated with $L\left(B_{L}, \mathrm{~b}\right)$ and $L_{0}\left(B_{L_{0}}, \mathrm{c}\right)$. The stretch modulus is also calculated for the central three DNA turns associated with $L, L_{0}$, and the corresponding additive helical parameters, ${ }^{12}$ shift, slide and rise (d, e). The average values and their standard deviations were calculated using all possible subframents with a particular length.

We now turn to the effects of electrostatic repulsion on the stretching response of DNA. 
The derived variations from MD trajectories for both normal and neutral DNA helices exhibit a nearly linear dependence on contour length $\left(L_{0}\right)$, and a faster-than-linear growth for the end-to-end distance ( $L$, Figure 2a), in accord with previous simulation results of Noy and Golestanian. ${ }^{12}$ Although the phosphate neutralization greatly enhances the fluctuation of the end-to-end distance, indicated by the larger variance, the variance profiles in terms of contour length differ slightly. This difference arises because, rather than the essentially uncorrelated and independent behaviour of contour length, the end of the duplex fluctuates in a cooperative way and can be influenced by the motions of neighbouring segments, ${ }^{9,12}$ which are affected by the backbone electrostatic interactions. Further examination indicates lower variances for the contour length on length scales over three helical turns for neutral DNA compared to its normal counterpart. The smaller variances of $L_{0}$ for neutral DNA at long length scales imply enhanced rigidity because of the phosphate neutralization. Correspondingly, neutral DNA exhibits a significantly larger stretching modulus $\left(B_{L_{0}}\right)$ than normal DNA on all length scales (Figure 2c). For the end-to-end distance, the enhanced superlinear growth in the variance with respect to the number of bases at long length scales suggests that phosphate neutralization contributes to the stretching cooperativity for DNA duplexes longer than one to two helical turns. Hence the effects of backbone electrostatic interactions on the stretching fluctuations are length scale dependent. The elastic modulus exhibits a slightly larger value for neutral DNA from one base pair to approximately two DNA turns, above which an enhanced softening was observed, finally resulting in smaller $B_{L}$ values by about $250 \mathrm{pN}$ compared with the normal charged form (Figure $2 \mathrm{~b}$ ). This result indicates the reduced vibrations thus weakened stretching cooperativity at lengths less than two DNA turns because of the stronger stacking interactions when backbone phosphates are neutralized.

The above results raise the question of why end-to-end distance and contour length stretch definitions with respect to the duplex length exhibit such different elastic responses to phosphate neutralization. To exclude the end effects on the local elastic properties of 
inter-base pairs, we calculate the stretch modulus of the longitudinal rise, and the two lateral shear components (i.e., slide and shift) of the central three helical turns of the 63mer oligonucleotide (Figure 2d,e). We find that the neutral DNA produces increased force constants for the three helical parameters compared with normal DNA, especially for the rise parameter (displacement along the helix axis), where the elastic modulus increases by about $1500 \mathrm{pN}$. Since the effective elastic modulus of contour length can be roughly quantified using the average value of the three local force constants, ${ }^{12}$ it is understandable that the stiffness of contour length is significantly enhanced after phosphate neutralization. We then analysed the behaviour of end-to-end distance for the central three helical turns of our model DNA helix strand, and found that $B_{L}$ first increases from a value of approximately $1500 \mathrm{pN}$ to a maximum at around $8 \mathrm{bp}$, and finally reaches a plateau (around $1300 \mathrm{pN}$ ). This result accords with the values previously reported in simulations ${ }^{12,41}$ and experiments. ${ }^{42}$ The stiffening of the end-to-end distance for DNA below two helical turns after phosphate neutralization is retained. This result occurs because for short duplexes, the end-to-end distance is predominantly influenced by the rise parameter at short length scales. ${ }^{12}$ The larger modulus of the rise, caused by the stronger stacking interactions upon backbone neutralization, explains why $B_{L}$ exhibits a relative stiffening on short length scales. Similar to the normal DNA, evidence of cooperativity with a crossover at around one DNA turn was observed after neutralization, confirming the critical role of end effects correlating with the stretching cooperativity and the soft stretch modulus of DNA on long length scales. ${ }^{12,20}$ It is interesting to note that elastic constants for the end-to-end distance at the plateau stage are very close to each other in the internal fragment for neutral and normal 63-mer DNAs. The negligible effects of backbone repulsion are in agreement with overstretching experiments of single DNA molecules, which revealed a stretch modulus of around $1300 \mathrm{pN}$, with a weak dependence on ion concentration. ${ }^{43}$ These results verify the crucial role of cooperative motions in determining the behaviour of DNA stretching on length scales beyond three helical turns. 

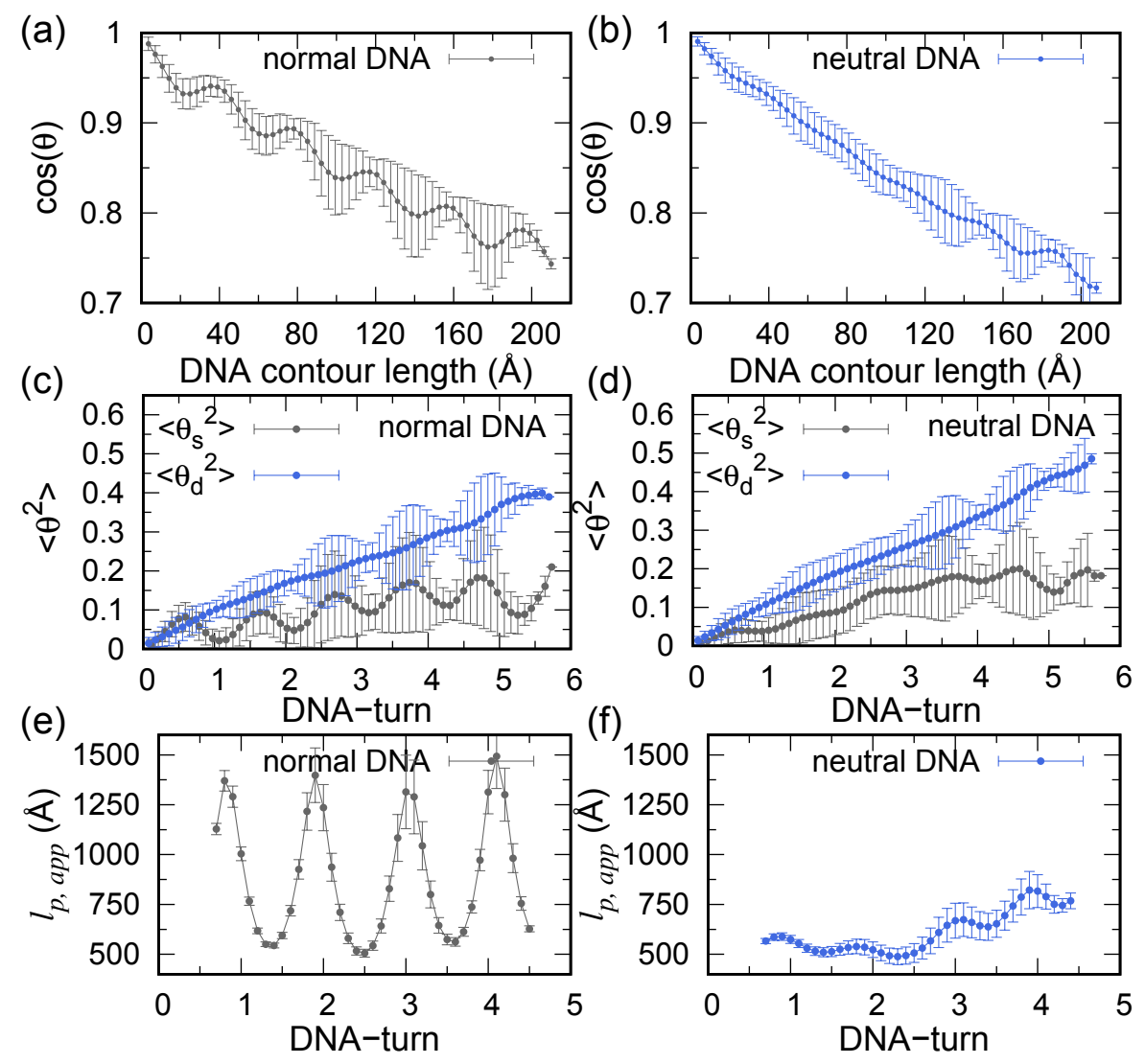

Figure 3: The cosine functions between base-pair axial vectors derived from MD trajectories in normal (a) and neutral (b) DNA systems. (c) and (d) show the static and dynamic contributions to the fluctuations of the bending angle $\theta$. (e) and (f) present the apparent persistence lengths $\left(l_{p, a p p}\right)$ calculated by a linear fit of the local directional decay, and the error bars are the asymptotic standard errors of the fitted persistence length. ${ }^{12}$ 

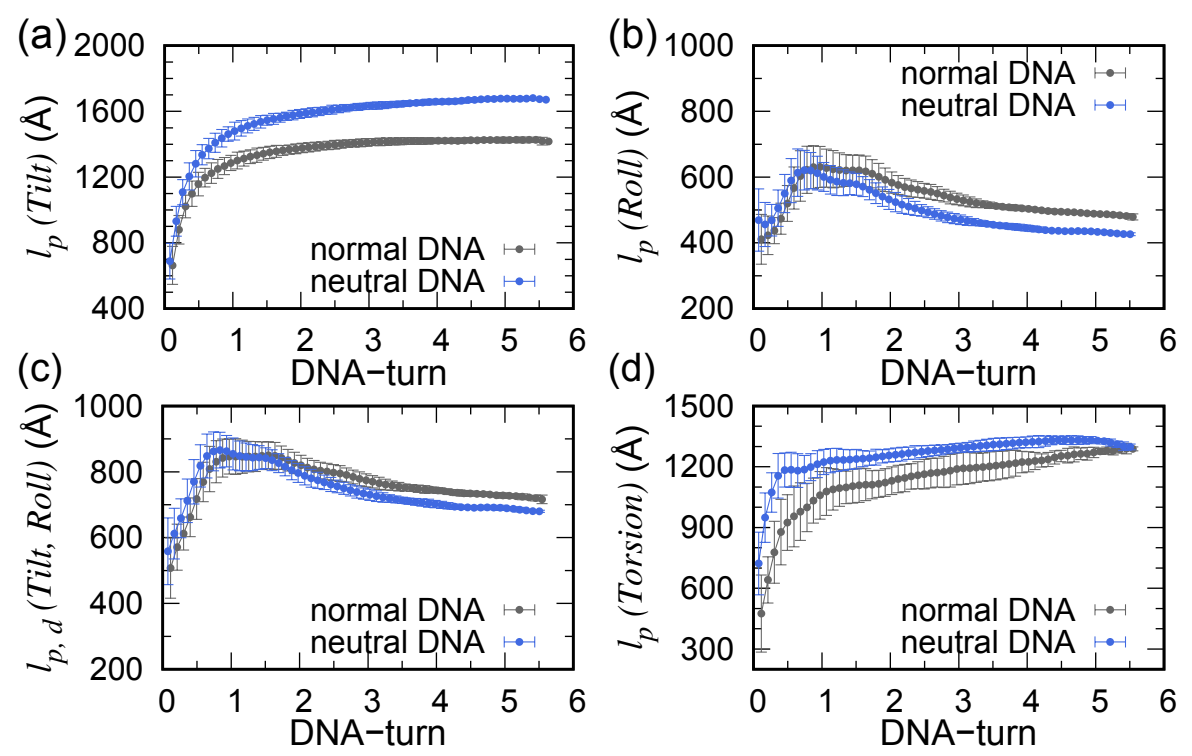

Figure 4: The persistence length $\left(l_{p}\right)$ contributions of tilt (a), and roll (b), and the dynamic persistence length contributed by tilt and roll (c) calculated from the MD trajectories. (d) is the torsional persistence length.

Figures 3a,b show the chain length dependence of directional decay for the 63-mer derived from MD trajectories. Rather than the uniform decay predicted by the traditional WLC model, DNA in its true fully charged state exhibits periodic oscillations, with the helical period for directional correlation of local bending, in accord with previous experimental and simulation results. ${ }^{12,34,44}$ The orientation of the chain end moves rapidly away from the normal vector of the reference Cartesian frame at the chain origin for fragment lengths ranging from $n$ to $n+\frac{1}{2}$ helical turns, and recovers for lengths between $n+\frac{1}{2}$ and $n+1$ turns. In particular, the profiles of $\langle\cos \theta\rangle$ exhibit almost the same amplitude of deviation from linearity. In contrast to the bending periodicity of normal DNA, neural DNA exhibits a nearly uniform and linear growth in the bending correlation with respect to increasing lengths for fragments less than five helical turns, and weak oscillations beyond. In the wedge theory of DNA curvature, bends are classified as static $\left(\theta_{s}\right)$ and dynamic $\left(\theta_{d}\right)$, and these two contributions correlate with the bending angle fluctuations as $\left\langle\theta^{2}\right\rangle=\left\langle\theta_{s}^{2}\right\rangle+\left\langle\theta_{d}^{2}\right\rangle$, 
where the angle brackets indicate averaging over all possible dinucleotide steps, and bending angles. Both normal and neutral oligonucleotides exhibit a net linear increase in $\left\langle\theta_{d}^{2}\right\rangle$ with fragment length, which yields 103 and $86 \mathrm{~nm}$ for the dynamic persistence length $\left(l_{p, d}\right)$, respectively. These values for the dynamic persistence length agree with single molecule experiments $\left(l_{p, d}=70 \sim 110 \mathrm{~nm}\right.$ as the concentration of $\mathrm{Na}^{+}$ranges from 10.0 to $\left.1.0 \mathrm{mM}\right) .{ }^{3}$ However, the periodicity observed in the static bending correlations of fully charged DNA is greatly reduced after phosphate neutralization (Figure 3c,d). This linear growth in the bending correlation of neutral DNA at lengths shorter than five helical turns agrees with the conventional description of the DNA duplex as an ideal elastic model. Furthermore, the pronounced variations in apparent persistence length, which is calculated using the local definition of short segments ${ }^{12}$ with $16 \mathrm{bp}$, is significantly reduced on phosphate neutralization (Figure 3e,f). The loss of periodicity indicates the crucial role of electrostatic interactions between backbone phosphates in the cooperative fluctuations and orientational memories between neighbouring steps.

At the structural level, DNA bending mainly arises from the successive rotation in terms of tilt $(\tau)$ and roll $(\rho)$. For harmonic bending potentials, the variances of roll and tilt are related to the dynamic persistence length by $2 L_{0} / l_{p, d}=\sigma_{\tau}^{2}+\sigma_{\rho}^{2}$, where $\sigma^{2}$ represents the variance. Using this formulation, dynamic persistence lengths with respect to fragment lengths were calculated (Figure 4c). We see that $l_{p, d}$ increases monotonically to the crossover at one helical turn from around $50 \mathrm{~nm}$ at the level of single base pair, then softens by approximately one third, and finally gives a plateau value of around $75 \mathrm{~nm}$ beyond four turns. The persistence lengths in the plateau regions are 75 and $70 \mathrm{~nm}$ for the normal and neutral DNA duplexes, respectively. This trend agrees with the results calculated using dynamic bending correlations, showing that the DNA molecules become less stiff as the charge is reduced. This result is consistent with the elasticity of single DNA molecules as a function of ionic strength, ${ }^{3}$ where $l_{p, d}$ is smaller for stronger ionic strength because of increased electrostatic screening. The persistence lengths of roll exhibit similar trends 
(Figure 4b), confirming that DNA bending flexibility is mostly due to changes in the roll angle, ${ }^{41}$ which is preferentially involved in spontaneous bending. In contrast, $l_{p, d}$ for tilt in neutral DNA is larger than that of normal DNA at all length scales (Figure 4a).

Similar calculations were performed for torsion flexibility, revealing a crossover at approximately one helical turn, in line with the simulations of Noy and Golestanian. ${ }^{12}$ The calculated torsional modulus is around $50 \mathrm{~nm}$ at the single base-pair level, and gives a large-scale asymptotic value of around $130 \mathrm{~nm}$, which is consistent with single-molecule experiments (around $120 \mathrm{~nm}$ ). ${ }^{45-47}$ The slight deviation may be attributed to the different DNA sequences as well as salt concentrations and applied forces in experiments. ${ }^{46,48}$ The neutral duplex has larger values for the torsional persistence length compared to normal DNA at all scales. Interestingly, the difference in the persistence length between these two forms of DNA decreases as the fragment elongates, suggesting that phosphate neutralization affects the DNA torsion mainly for a couple of DNA turns, but vanishes beyond. For angular parameters, phosphate neutralization increases the DNA rigidity for fragments shorter than a full turn, but has the opposite effect for roll and for tilt or torsion at long length scales (Figure 4). These distinct behaviours of roll and tilt or torsion rigidities as a function of length scale support the conclusion that the electrostatic contribution to the DNA rigidity is length scale dependent. For the smaller rise value after neutralization (see Table S2), we suspect that the electrostatic repulsion between backbone counteracts internal compressive base stacking to some extent, and therefore softens the conformational rigidity at short length scales, leading to the large flexibility for short oligonucleotides observed in single molecule experiments. ${ }^{11}$ On long length scales, B-DNA bends most easily in a groove direction through roll angle, i.e., bending anisotropy, while tilt and torsion make no net contribution and correlate more with the mechanical properties of local base pair steps. ${ }^{49}$ It is not surprising that roll persistence length gives very similar profiles to the end-to-end distance for normal and neutral DNA strands.

The last question is the contribution of electrostatics to the net bending persistence 
length. We use the variation of DNA persistence length on phosphate neutralization to estimate the electrostatic contribution. The net persistence lengths are obtained using overall average slope with respect to bending angle, shown in Figure 3a,b, are 74 and $67 \mathrm{~nm}$ for the normal and neutral DNA duplex, respectively. These results suggest a contribution of around $9 \%$ for the electrostatic persistence length. The persistence length can also be obtained by combining static and dynamic persistence lengths using $1 / l_{p}=1 / l_{p, s}+1 / l_{p, d}$. The static bending correlation (see Figure 3c,d) yields $l_{p, s}=236$ and $184 \mathrm{~nm}$ for normal and neutral duplexes, respectively. The dynamic persistence length can be evaluated from the variances of tilt and roll (Figure 4c), neglecting the contribution from twist, giving persistence lengths of 57 and $51 \mathrm{~nm}$ for normal and neutral strands, respectively. This analysis suggests a contribution of around $12 \%$ for the electrostatics. Our calculations suggest a less important role for DNA electrostatics in governing stiffness, and support the theoretical prediction of Odijk and Skolnick and Fixman (OSF), ${ }^{50,51}$ that the electrostatic repulsion contributes less than $10 \%$ to the total persistence length, with base pair stacking interactions dominate the stiffness of DNA. The persistence length derived using the variances of roll and tilt is not strictly the true value, since all six step degrees of freedom contribute to DNA bending because of local distortions. Note that because the deviations from the ideal BDNA helix remain small, ${ }^{52}$ we can still use the dynamic persistence length derived using the variances of roll and tilt to evaluate the net persistence length. We note that the bending flexility correlates strongly with the DNA sequence and the type of metallic ion in solution. ${ }^{53}$ Although the calculated bending stiffness here is slightly larger than the widely used bare persistence length of $50 \mathrm{~nm}$ for DNA, it still falls in the range of experimental observations. $^{3,4,43,53}$ 


\section{Conclusions}

In summary, we find that the intrinsic bending dynamics for short and intermediate lengths of DNA deviate from ideal harmonic elastic rods. In contrast, the neutral "null isomer" can be accurately described by the ideal WLC model, exhibiting a uniform decay for the directional correlation of local bending, rather than the helical periodicity observed in the fully charged oligonucleotide. The backbone neutralization softens the stretch modulus measured by end-to-end distance at all scales, except for fragments shorter than one to two full DNA turns. For the torsional persistence length, a monotonic crossover is observed for both the normal and neutral DNA isomers. However, the difference due to backbone net charges decreases with elongation of the DNA fragments. Our results demonstrate that phosphate neutralization increases the stiffness of DNA below two helical turns, but decreases it for longer strands, suggesting a length dependence for the contribution of electrostatic repulsion arising from negative net charges on the backbone to the DNA mechanics of bending. Backbone neutralization leads to softer vibrations, and hence weaker stretching cooperativity at lengths less than two DNA turns because of the stronger stacking interactions, but enhances the stretching cooperativity for longer strands. Furthermore, the proposed model can be used to evaluate the relative contribution of electrostatic and nonelectrostatic interactions to the persistence length. Our calculations indicate that base pair stacking dominates DNA

rigidity, in agreement with the OSF prediction. ${ }^{50,51}$ Our study contributes to the interpretation of DNA mechanics, DNA-protein interactions, DNA looping, and other key biophysical processes.

\section{Acknowledgement}

We thank the National Natural Science Foundation of China (Grant Nos. 91427304, 21434007, 21574122, and 51573175), and the Supercomputing Center of University of Science and Technology of China for computational source. 


\section{Supporting Information Available}

Simulation methods, the calculations of elastic modulus and the local base pair step parameters. This material is available free of charge via the Internet at http://pubs.acs.org/.

\section{References}

(1) Blackwood, E. M.; Kadonaga, J. T. Going the Distance: A Current View of Enhancer Action. Science 1998, 281, 60-63.

(2) Wong, O. K.; Guthold, M.; Erie, D. A.; Gelles, J. Interconvertible Lac Repressor-DNA Loops Revealed by Single-Molecule Experiments. PLoS Biol. 2008, 6, e232.

(3) Smith, S.; Finzi, L.; Bustamante, C. Direct Mechanical Measurements of the Elasticity of Single DNA Molecules by Using Magnetic Beads. Science 1992, 258, 1122-1126.

(4) Baumann, C. G.; Smith, S. B.; Bloomfield, V. A.; Bustamante, C. Ionic Effects on the Elasticity of Single DNA Molecules. Proc. Natl. Acad. Sci. U S A 1997, 94, 6185-6190.

(5) Bustamante, C.; Smith, S. B.; Liphardt, J.; Smith, D. Single-molecule Studies of DNA Mechanics. Curr. Opin. Struct. Biol. 2000, 10, 279-285.

(6) Cloutier, T. E.; Widom, J. Spontaneous Sharp Bending of Double-Stranded DNA. Mol. Cell 2004, 14, $355-362$.

(7) Cloutier, T. E.; Widom, J. DNA Twisting Flexibility and the Formation of Sharply Looped Protein-DNA Complexes. Proc. Natl. Acad. Sci. USA 2005, 102, 3645-3650.

(8) Wiggins, P. A.; van der Heijden, T.; Moreno-Herrero, F.; Spakowitz, A.; Phillips, R.; Widom, J.; Dekker, C.; Nelson, P. C. High Flexibility of DNA on Short Length Scales Probed by Atomic Force Microscopy. Nat. Nanotechnol. 2006, 1, 137-141. 
(9) Mathew-Fenn, R. S.; Das, R.; Harbury, P. A. B. Remeasuring the Double Helix. Science 2008, 322, 446-449.

(10) Yuan, C.; Chen, H.; Lou, X. W.; Archer, L. A. DNA Bending Stiffness on Small Length Scales. Phys. Rev. Lett. 2008, 100, 018102.

(11) Vafabakhsh, R.; Ha, T. Extreme Bendability of DNA Less than 100 Base Pairs Long Revealed by Single-Molecule Cyclization. Science 2012, 33\%, 1097-1101.

(12) Noy, A.; Golestanian, R. Length Scale Dependence of DNA Mechanical Properties. Phys. Rev. Lett. 2012, 109, 228101.

(13) Yan, J.; Marko, J. F. Localized Single-Stranded Bubble Mechanism for Cyclization of Short Double Helix DNA. Phys. Rev. Lett. 2004, 93, 108108.

(14) Wiggins, P. A.; Phillips, R.; Nelson, P. C. Exact Theory of Kinkable Elastic Polymers. Phys. Rev. E 2005, 71, 021909.

(15) Le, T. T.; Kim, H. D. Probing the Elastic Limit of DNA Bending. Nucleic Acids Res. 2014, 42, 10786.

(16) Du, Q.; Smith, C.; Shiffeldrim, N.; Vologodskaia, M.; Vologodskii, A. Cyclization of Short DNA Fragments and Bending Fluctuations of the Double Helix. Proc. Natl. Acad. Sci. USA 2005, 102, 5397-5402.

(17) Harrison, R. M.; Romano, F.; Ouldridge, T. E.; Louis, A. A.; Doye, J. P. K. Coarse-grained modelling of strong DNA bending II: Cyclization. arXiv e-prints 2015, arXiv:1506.09008.

(18) Vologodskii, A.; D. Frank-Kamenetskii, M. Strong Bending of the DNA Double Helix. Nucleic Acids Res. 2013, 41, 6785-6792.

(19) Becker, N. B.; Everaers, R. Comment on "Remeasuring the Double Helix". Science 2009, 325, 538-538. 
(20) Wu, Y.-Y.; Bao, L.; Zhang, X.; Tan, Z.-J. Flexibility of Short DNA Helices With Finite-length Effect: From Base Pairs to Tens of Base Pairs. J. Chem. Phys. 2015, $142,125103$.

(21) Mazur, A. K.; Maaloum, M. DNA Flexibility on Short Length Scales Probed by Atomic Force Microscopy. Phys. Rev. Lett. 2014, 112, 068104.

(22) Mazur, A. K.; Maaloum, M. Atomic Force Microscopy Study of DNA Flexibility on Short Length Scales: Smooth Bending Versus Kinking. Nucleic Acids Res. 2014, 42, 14006-14012.

(23) Peters, J. P.; Maher, L. J. DNA Curvature and Flexibility in Vitro and in Vivo. $Q$. Rev. Biophys. 2010, 43, 23-63.

(24) Nomidis, S. K.; Kriegel, F.; Vanderlinden, W.; Lipfert, J.; Carlon, E. Twist-Bend Coupling and the Torsional Response of Double-Stranded DNA. Phys. Rev. Lett. 2017, $118,217801$.

(25) Skoruppa, E.; Nomidis, S. K.; Marko, J. F.; Carlon, E. Bend-Induced Twist Waves and the Structure of Nucleosomal DNA. Phys. Rev. Lett. 2018, 121, 088101.

(26) Manning, G. S. The Persistence Length of DNA Is Reached from the Persistence Length of Its Null Isomer through an Internal Electrostatic Stretching Force. Biophys. J. 2006, 91, 3607-3616.

(27) Kosikov, K. M.; Gorin, A. A.; Lu, X.-J.; Olson, W. K.; Manning, G. S. Bending of DNA by Asymmetric Charge Neutralization: All-Atom Energy Simulations. J. Am. Chem. Soc. 2002, 124, 4838-4847.

(28) Savelyev, A.; Materese, C. K.; Papoian, G. A. Is DNA's Rigidity Dominated by Electrostatic or Nonelectrostatic Interactions? J. Am. Chem. Soc. 2011, 133, 19290-19293. 
(29) Xiao, S.; Zhu, H.; Wang, L.; Liang, H. DNA Conformational Flexibility Study Using Phosphate Backbone Neutralization Model. Soft Matter 2014, 10, 1045-1055.

(30) Kahn, J. D. DNA, Flexibly Flexible. Biophys. J. 2014, 107, 282-284.

(31) Lu, X.-J.; Hassan, M. E.; Hunter, C. Structure and Conformation of Helical Nucleic Acids: Analysis Program (SCHNAaP). J. Mol. Biol. 1997, 273, 668-680.

(32) Olson, W. K. et al. A Standard Reference Frame for the Description of Nucleic Acid Base-pair Geometry. J. Mol. Biol. 2001, 313, 229-237.

(33) Lu, X.-J.; Olson, W. K. 3DNA: A Software Package for the Analysis, Rebuilding and Visualization of Three-dimensional Nucleic Acid Structures. Nucleic Acids Res. 2003, 31,5108 .

(34) Mazur, A. K. Evaluation of Elastic Properties of Atomistic DNA Models. Biophys. J. 2006, $91,4507-4518$.

(35) Mazur, A. K. Wormlike Chain Theory and Bending of Short DNA. Phys. Rev. Lett. 2007, 98, 218102.

(36) Whitley, K. D.; Comstock, M. J.; Chemla, Y. R. Ultrashort Nucleic Acid Duplexes Exhibit Long Wormlike Chain Behavior with Force-Dependent Edge Effects. Phys. Rev. Lett. 2018, 120, 068102.

(37) Ma, N.; van der Vaart, A. Anisotropy of B-DNA Groove Bending. J. Am. Chem. Soc. 2016, 138, 9951-9958.

(38) Kamashev, D. E.; Mazur, A. K. Single-Stranded Breaks Relax Intrinsic Curvature in DNA? Biochemistry 2004, 43, 8160-8168.

(39) LankaS, F.; Sponer, J.; Langowski, J.; III, T. E. C. DNA Base pair Step Deformability Inferred from Molecular Dynamics Simulations. Biophys. J. 2003, 85, 2872-2883. 
(40) Norouzi, D.; Mohammad-Rafiee, F.; Golestanian, R. Effect of Bending Anisotropy on the 3D Conformation of Short DNA Loops. Phys. Rev. Lett. 2008, 101, 168103.

(41) Spiriti, J.; Kamberaj, H.; de Graff, A. M. R.; Thorpe, M. F.; van der Vaart, A. DNA Bending through Large Angles Is Aided by Ionic Screening. J. Chem. Theory Comput. 2012, 8, 2145-2156.

(42) Wang, M.; Yin, H.; Landick, R.; Gelles, J.; Block, S. Stretching DNA With Optical Tweezers. Biophys. J. 1997, 72, $1335-1346$.

(43) Wenner, J. R.; Williams, M. C.; Rouzina, I.; Bloomfield, V. A. Salt Dependence of the Elasticity and Overstretching Transition of Single DNA Molecules. Biophys J. 2002, 82, $3160-3169$.

(44) Maroun, R. C.; Olson, W. K. Base Sequence Effects in Double-helical DNA. III. Average Properties of Curved DNA. Biopolymers 1988, 27, 585-603.

(45) Moroz, J. D.; Nelson, P. Torsional Directed Walks, Entropic Elasticity, and DNA Twist Stiffness. Proc. Natl. Acad. Sci. USA 1997, 94, 14418-14422.

(46) Kriegel, F.; Ermann, N.; Forbes, R.; Dulin, D.; Dekker, N. H.; Lipfert, J. Probing the Salt Dependence of the Torsional Stiffness of DNA by Multiplexed Magnetic Torque Tweezers. Nucleic Acids Res. 2017, 45, 5920-5929.

(47) Lipfert, J.; Kerssemakers, J. W. J.; Jager, T.; Dekker, N. H. Magnetic Torque Tweezers: Measuring Torsional Stiffness in DNA and RecA-DNA Filaments. Nat. Methods 2010, 7, $977-980$.

(48) Shi, X.; Herschlag, D.; Harbury, P. A. B. Structural Ensemble and Microscopic Elasticity of Freely Diffusing DNA by Direct Measurement of Fluctuations. Proc. Natl. Acad. Sci. USA 2013, 110, E1444-E1451. 
(49) Zhurkin, V. B.; Ulyanov, N. B.; Gorin, A. A.; Jernigan, R. L. Static and Statistical Bending of DNA Evaluated by Monte Carlo Simulations. Proc. Natl. Acad. Sci. U S A 1991, 88, 7046-7050.

(50) Odijk, T. Polyelectrolytes Near the Rod Limit. J. Polym. Sci. Polym. Phys. Ed. 1977, $15,477-483$.

(51) Skolnick, J.; Fixman, M. Electrostatic Persistence Length of a Wormlike Polyelectrolyte. Macromolecules 1977, 10, 944-948.

(52) Meyer, S.; Jost, D.; Theodorakopoulos, N.; Peyrard, M.; Lavery, R.; Everaers, R. Temperature Dependence of the DNA Double Helix at the Nanoscale: Structure, Elasticity, and Fluctuations. Biophys. J 2013, 105, 1904-1914.

(53) Guilbaud, S.; Salomé, L.; Destainville, N.; Manghi, M.; Tardin, C. Dependence of DNA Persistence Length on Ionic Strength and Ion Type. Phys. Rev. Lett. 2019, 122, 028102. 